\title{
Evaluación de Resultados e Impactos de un Modelo de Salud Mental basado en la Comunidad en Localidades de Bogotá, DC
}

\author{
Luis Jorge Hernández F. \\ Médico. M. Sc. Salud Pública. Especialista en Administración en Salud. Secretaría Distrital de Salud de \\ Bogotá. E-mail: ljhf@hotmail.com
}

\section{RESUMEN}

Objetivos Se evaluaron los resultados e impactos de la primera fase de un modelo de salud mental basado en la comunidad, desarrollado en la Red Sur por la Secretaria de Salud de Bogotá y el Hospital de Tunjuelito, durante el año 2002. La primera fase del programa consistió en la formación de agentes comunitarios en salud, realización de tamizajes comunitarios de salud mental mediante visita domiciliaria, remisiones a la red de servicios y aumento de nodos de la red de buen trato.

Métodos Se compararon los indicadores de antes (año 2001) y después (año 2002) de la aplicación del modelo y las variaciones entre los grupos de estudio (Red Sur) y de control (Red Centro-Oriente).

Resultados El modelo de salud mental incrementó la notificación de casos de violencia intrafamiliar (23,8 \% grupos de estudio vs. $15,3 \%$ grupo control), el seguimiento de casos de violencia intrafamiliar ( $80 \%$ vs. $25 \%$ ) y la conectividad en las redes de buen trato, valorada por la proporción de nodos comunitarios (39,2\% vs. 30,0 \%). Así mismo, se identificó que en las localidades donde el componente de salud mental del Plan de Atención Básica-PAB está más fortalecido, el modelo de salud mental presentó mejor desempeño.

Conclusiones Los resultados sugieren que la realización de un modelo de salud mental basado en la comunidad favorece la notificación de casos de violencia intrafamiliar, el seguimiento de los mismos y una mayor composición comunitaria en las redes sociales de buen trato.

Palabras Claves: Salud mental, estudios de evaluación, evaluación de programas, evaluación de impacto (fuente: DeCS, BIREME) 


\section{ABSTRACT \\ Evaluation of results and impact of the first phase of a Community Based Mental Health Model in localities in Bogotá, D.C.}

Objectives The results and impact of the first phase of a Community Based Mental Health Model, developed in the South network by the Bogota Health authority and the Tunjuelito hospital during 2002 were evaluated. The first phase of the program included the formation of health community agents, communitary screening of mental health done through home visits, referrals to the services network and an increase in the nodes of the network of good treatment.

Methods The indicators of before (year 2001) and after (year 2002) the application of the model were compared, as well as the variations between the study groups (South network) and the control group (Central-Eastern network).

Results The mental health model increased the notification of cases of family violence $(23,8 \%$ in the study group vs. $15,3 \%$ in the control group), the followup of cases of family violence (80 \% vs. $25 \%)$ and the conectivity of the networks of good treatment. In the localities in which the mental health component of the Basic Attention Plan is strengthened, the mental health model showed a better performance.

Conclusions The results suggest that the implementation of a community based mental health model favors the notification of cases of family violence, their followup and a greater community composition in the social networks of good treatment.

Key Words: Mental health, evaluation studies, program evaluation, (source: DeCS, BIREME)

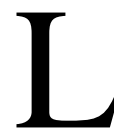

a investigación evaluativa o la "investigación de evaluación”, como la describe Polit tiene un carácter "utilitario", es decir pretende "dar respuestas prácticas a los responsables de la toma de decisiones”, en ese sentido es también evaluación de políticas en salud (1).

El Banco Mundial ha establecido que la evaluación de impacto tiene como objetivo determinar si el programa, plan o proyecto “produjo los efectos deseados en las personas, hogares e instituciones y si esos efectos son atribuibles a la intervención del programa. Las evaluaciones de impacto también permiten examinar consecuencias no previstas en los beneficiarios, ya sean positivas o negativas"(2).

La OPS ha señalado que en el idioma español la palabra "evaluación” se utiliza indistintamente para tres intervenciones: evaluación, seguimiento y evaluación ex post.

La evaluación ex ante valora la viabilidad (política y ética) y factibilidad (financiera, técnica y administrativa) de una propuesta de proyecto. El monitoreo se realiza "durante” la ejecución de un proyecto y se realiza para garantizar que se cumplan los objetivos y metas propuestas en el mismo, lo cual supone que el monitoreo permite detectar tempranamente "desviaciones" para formular correctivos oportunos( 3,4$)$. 
La evaluación ex post se realiza una vez finalizado el proyecto y se considera que lo deben realizar las personas que hacen la formulación del lineamiento político y realizan función decisoria. En esta evaluación se valoran el cumplimiento de metas y objetivos y sé retroalimenta el diseño de fases ulteriores o de otros proyectos similares. La OPS enfatiza en que en esta fase se proponen intervenciones para mejorar los procesos de cooperación para la realización del proyecto $(5,6)$.

La evaluación ex post parte de un presupuesto o premisa importante: Que el proyecto se haya diseñado con un "enfoque de marco lógico", es decir, que los objetivos y metas estén adecuadamente formulados. Se puede inferir que una meta "bien formulada", está planteada en términos de resultados cuantificables en el nivel de salud de las personas o de sus determinantes, es decir en términos de impacto.

La Guía Merseyside para evaluación de impacto establece que la evaluación de impacto en salud es la estimación de los efectos de una acción especifica en la salud de una población determinada $(7,8)$. Indica que la evaluación de impacto en salud debe ser prospectiva y tiene dos propósitos:

- Evaluar el potencial impacto en salud, positivo o negativo de programas, proyectos y políticas.

- Mejorar la calidad de las políticas públicas a través de recomendaciones que permitan aumentar los impactos positivos y minimizar los negativos.

La guía parte de la conceptualización de impacto en salud como “...un cambio en el estado de salud (o en los determinantes del estado en salud) de un individuo o grupo atribuidos a programas, proyectos o políticas...", y define los determinantes de salud como un factor conocido que tiene un potencial para producir cambios en el estado de salud. El concepto de determinantes que se utiliza en la mencionada guía es el de modelo socio-ambiental de salud elaborado por Lalonde y Labonté (8). Establece así mismo, que el proceso de evaluación de impacto en salud requiere amplia participación, la cooperación de expertos, de un amplio rango de "stakeholders" (grupos de interés y personas quienes han sido involucradas en el proyecto o podrían ser directamente afectadas por este) e informantes claves (personas cuyas funciones o roles se producen a partir del conocimiento o información de relevancia del proyecto y sus resultados). Es esencial la participación pública o comunitaria a lo largo de la evaluación de impacto en salud (9).

La primera fase del modelo de salud mental en Bogotá

El modelo de salud mental basado en la comunidad constituye realmente un modelo de atención en salud, y corresponde a una forma de atención primaria en salud y no es solamente una forma de desinstitucionalizar a los pacientes mentales (10).

El modelo de salud mental basado en la comunidad en su primera fase se desarrolló a través de las siguientes intervenciones: 
- Formación en la Red Sur de 150 personas como agentes comunitarios en salud con énfasis en salud mental, las cuales están en capacidad de realizar identificación, promoción y refuerzo de los factores de resiliencia individual, familiar, social y cultural, y de movilización social alrededor de la salud mental. Esta movilización alrededor de la salud mental se entiende como mayor giro alrededor de un eje problemático como es la violencia intrafamiliar y sexual.

- Tamizajes comunitarios de factores de riesgo (disfunción familiar, niveles de estrés, aislamiento social) y de evento (violencia intrafamiliar, abuso sexual, uso de psicoactivos y conducta suicida). Durante el año 2002 se realizaron 361 tamizajes comunitarios de salud mental y 100 canalizaciones a la red de servicios. La mayoría de personas canalizadas tienen calidad de vinculados al sistema de seguridad social en salud y se buscó que consultaran al servicio de salud mental, facilitando el acceso a través de la información y orientación explicita al servicio de psiquiatría. Un aumento en la consulta externa de psiquiatría se interpretó como una mayor movilización comunitaria alrededor de la salud mental.

- Formulación de los programas de intervención sistémica extramural orientados a la transformación de los diferentes contextos (familiar, institucional, laboral) que influyeron en los patrones de comportamiento individuales.

- Fortalecimiento de las redes sociales y comunitarias como la red de buen trato de las localidades, para la inclusión del individuo en los diferentes espacios sociales.

- Visitas domiciliaria que incluyeron la realización de ecomapa y apgar familiar , y que reforzaron las actividades extramurales de promoción de los factores de resiliencia individual y familiar, así como la autonomía de las familias y comunidades para el aprovechamiento de sus recursos a favor de la salud mental de cada uno de los integrantes de la familia.

Se realizó la evaluación del resultado e impacto de la primera fase del modelo salud mental basado en la comunidad implementado en las localidades de influencia de la Red Sur, durante el año 2002.

\section{MATERIALES Y METODOS}

Se realizó un estudio de evaluación de resultados e impacto por medio de las comparaciones antes (año 2001)-después (año 2002), y del grupo estudio (Red Sur) con respecto al grupo control (Red Centro-Oriente) (11).

El grupo de estudio se conformó con las localidades de la Red Sur: Ciudad Bolívar, Usme, Rafael Uribe Uribe, Antonio Nariño y Tunjuelito. En esta red se realizó durante el año 2001 la primera fase del modelo de salud mental basado en la comunidad. No se consideró para esta 
investigación la localidad de Sumapaz ya que por ser una localidad exclusivamente rural el abordaje en salud mental se hace por otras estrategias del Plan de Atención Básica.

El grupo control fue la Red Centro-Oriente conformada por las localidades de Candelaria, Mártires, Santa Fé y San Cristóbal.

Identificación de los descriptores e indicadores

Se conformó un grupo de trabajo integrado por representantes de la Red Sur, específicamente la Empresa Social del Estado-ESE u Hospital de Tunjuelito, que en conjunto con el grupo funcional de Salud Mental de la Secretaría Distrital de Salud, realizaron durante el año 2002 la dirección y operación de la primera fase del modelo de salud mental basado en la comunidad en la Red Sur. El grupo de trabajo realizó la reconstrucción analítica del problema que dio origen al proyecto, lo cual permite la identificación de los descriptores e indicadores que manifiestan la existencia del problema y la identificación de los factores que lo produjeron. Se identificaron cuatro descriptores con sus correspondientes factores críticos (Cuadro 1).

Cuadro 1. Descriptores y factores críticos en la primera fase del modelo de salud mental basado en la comunidad

\begin{tabular}{|c|c|}
\hline Descriptores & Factores criticos \\
\hline $\begin{array}{l}\text { 1. Modificación de la notificación de casos e } \\
\text { violencia intrafamiliar a través del SIVIM (Sis- } \\
\text { tema de Vigilancia epidemiológica de violencia } \\
\text { intra-familiar). La no notificación constituye un } \\
\text { problema en la medida que refleja la baja ca- } \\
\text { pacidad de evidenciar un evento que afecta la } \\
\text { salud pública tal como lo constituye la violen- } \\
\text { cia intrafamiliar y sexual. }\end{array}$ & $\begin{array}{l}\text { Se espera que el modelo de salud mental fa- } \\
\text { vorezca la mayor sensibilización en trabajado- } \\
\text { res de la salud de la red adscrita y comunidad } \\
\text { en general para que consulten por violencia } \\
\text { intrafamiliar y violencia sexual y que se evi- } \\
\text { dencie más casos que son notificados a través } \\
\text { del SIVIM. Se espera modificaciones en la no- } \\
\text { tificación de casos. }\end{array}$ \\
\hline $\begin{array}{l}\text { 2. Seguimiento de casos: A través del modelo } \\
\text { se busca aumentar el seguimiento de casos } \\
\text { de violencia intrafamiliar y violencia sexual, e } \\
\text { inicio de ruta critica a partir de la notificación. }\end{array}$ & $\begin{array}{l}\text { Notificación: A través de la ficha de vigilancia } \\
\text { epidemiológica de violencia intrafamiliar SI- } \\
\text { VIM. Los casos a los que se les hace segui- } \\
\text { miento son los notificados a través de este } \\
\text { instrumento. }\end{array}$ \\
\hline $\begin{array}{l}\text { 3. Red de buen Trato: A través del modelo se } \\
\text { busca consolidar la red es decir aumentar el } \\
\text { número de nodos institucionales y comunita- } \\
\text { rios. }\end{array}$ & $\begin{array}{l}\text { Aumento de nodos: Se refiere a la identifica- } \\
\text { ción de personas, entidades públicas o priva- } \\
\text { das que se constituyen en informantes clave o } \\
\text { grupos de interés en el eje problémico de vio- } \\
\text { lencia intra familiar y sexual y que se constitu- } \\
\text { yan en nodos de la red local de buen trato. }\end{array}$ \\
\hline $\begin{array}{l}\text { 4. Consulta externa de salud mental (Psiquia- } \\
\text { tría): A través del modelo de salud mental ba- } \\
\text { sado en la comunidad se generan canaliza- } \\
\text { ciones para que las personas consulten a los } \\
\text { servicios de salud mental de la red adscrita. }\end{array}$ & $\begin{array}{l}\text { Disponibilidad de servicios de salud mental, } \\
\text { consulta de psiquiatría. Para el grupo de tra- } \\
\text { bajo: las consultas de psiquiatría de la UPA } \\
\text { (unidad primaria de atención)San Benito en la } \\
\text { localidad de Tunjuelito y la UPA La Estrella en } \\
\text { Ciudad Bolívar. En el grupo control, consulta } \\
\text { de psiquiatría Hospital Centro-Oriente. }\end{array}$ \\
\hline
\end{tabular}


Con base en los descriptores anteriores se establecieron los indicadores a evaluar, y se identificaron los años de información disponible y los grupos de estudio y control y el tipo de evaluación a desarrollar (Cuadro 2).

Se utilizó estadística no paramétrica y la prueba exacta de Fisher para el análisis de resultados.

Los resultados de las comparaciones fueron presentados a un grupo focal conformado por las profesionales que realizaron la parte operativa de la primera fase del modelo de salud mental basado en la comunidad en la Red Sur para identificar posibles explicaciones a los mismos.

Cuadro 2. Indicadores, grupos y tipo evaluación

\begin{tabular}{|c|c|c|c|}
\hline $\begin{array}{l}\text { Indicador a partir del } \\
\text { Descriptor }\end{array}$ & $\begin{array}{l}\text { Grupo } \\
\text { Estudio }\end{array}$ & $\begin{array}{l}\text { Grupo } \\
\text { Control }\end{array}$ & Evaluación \\
\hline $\begin{array}{l}\text { Casos Notificados a través de } \\
\text { SIVIM años 2000, } 2001 \text { y } \\
2002 \text {. }\end{array}$ & Red Sur & $\begin{array}{l}\text { Red Centro- } \\
\text { Oriente }\end{array}$ & Resultado \\
\hline $\begin{array}{l}\text { Casos de violencia intrafami- } \\
\text { liar y violencia sexual segui- } \\
\text { dos a partir de los notificados } \\
\text { año } 2002 \text {. }\end{array}$ & Red Sur & $\begin{array}{l}\text { Red Centro- } \\
\text { Oriente }\end{array}$ & Resultado \\
\hline $\begin{array}{l}\text { Modificación en el número de } \\
\text { nodos y tipo de nodo en la } \\
\text { red de buen trato. El aumento } \\
\text { en el número y tipo de nodo } \\
\text { equivale a mayor conectividad } \\
\text { de la red. Años 2001-2002 }\end{array}$ & Red Sur & $\begin{array}{l}\text { Red Centro- } \\
\text { Oriente }\end{array}$ & Impacto \\
\hline $\begin{array}{l}\text { Consulta Externa de Salud } \\
\text { Mental, Consulta de Psiquia- } \\
\text { tría. Años 2001-2002 }\end{array}$ & $\begin{array}{l}\text { Red Sur: } \\
\text { Consultas de } \\
\text { Psiquiatría }\end{array}$ & $\begin{array}{l}\text { Red Centro- } \\
\text { Oriente: con- } \\
\text { sulta de psi- } \\
\text { quiatría Hos- } \\
\text { pital Centro- } \\
\text { Oriente }\end{array}$ & Resultado \\
\hline
\end{tabular}

\section{RESULTADOS}

Notificación de casos de violencia intrafamiliar a través de SIVIM

Al comparar por grupos se evidencia que antes del modelo tanto el grupo estudio (Red Sur) y el grupo control (Red Centro-Oriente) presentaron aumento de la notificación de casos a través del SIVIM en el periodo 2000-2001. Este aumento fue mayor en el grupo estudio en comparación con el grupo control (86,2 \% vs. 26,2 \%). Después de la intervención del modelo también se evidencia aumento en la notificación de los dos grupos, siendo más alta nuevamente en el grupo estudio (23,8 $\%$ vs. $15,3 \%)$. 
Sin embargo, al interior de la Red Sur las localidades que presentan mayor aumento de la notificación son: Antonio Nariño y Tunjuelito, las cuales muestran mayor crecimiento de la notificación durante la ejecución del modelo que antes de la implementación del mismo.

Seguimiento de casos de violencia intrafamiliar y violencia sexual

Al comparar por redes se evidencia que cuatro de las cinco las localidades de la Red Sur realizaron seguimiento al 100 por ciento de los casos frente a la Red Centro-Oriente en donde solamente una localidad logró el 100 por ciento de seguimientos a partir de la notificación.

Se resalta que las localidades de Antonio Nariño y Tunjuelito presentaron un mayor aumento en la notificación después del modelo y presentan también un 100 por ciento de cumplimiento en los seguimientos de casos de violencia intra-familiar y violencia sexual.

La proporción de localidades con más del 90 \% de seguimiento fue mayor en la Red Sur (80 \% vs. $25 \%$ ( $\mathrm{p}>0.05$ Test Fisher).

Conectividad red de buen trato

El grupo estudio (Red Sur) pasó de 56 nodos en el año 2001 a 84 nodos en el 2002, lo cual equivale al $50 \%$ de aumento en conectividad. Las localidades de este grupo que tuvieron mayor aumento en nodos son Tunjuelito que pasó de 6 a 14 nodos y Antonio Nariño que paso de 11 a 21 nodos.

La proporción de localidades con más del $50 \%$ de aumento en nodos de la red de buen trato fue mayor en la Red Centro-Oriente (50 \% vs. $40 \%$ )( p >0.05 Test Fisher).

Tipos de nodo en la Red de Buen Trato

En el grupo estudio, de los 28 nodos que ingresaron en el año 2002, 11 (39,2 \%) corresponden a entidades públicas; 6 (21,4 \%) a entidades privadas, ONG y académicas, y 11 nodos (39,3 \%) son organizaciones comunitarias. La localidad de Tunjuelito y Antonio Nariño presentaron el mayor aumento de nodos comunitarios.

El grupo estudio (Red Sur), presentó un 39,3 \% de nodos comunitarios frente a 30 \% de nodos comunitarios del grupo control (Red Centro Oriente). Sin embargo, al tener en cuenta en un solo grupo los nodos conformados por entidades privadas, ONG y académicas, en conjunto con los nodos comunitarios se evidencia que a esta categoría corresponden el 60,7 \% de los nodos del grupo estudio y el $45 \%$ de los nodos al grupo control. 
La proporción de nodos comunitarios fue mayor en las localidades donde se desarrollo la primera fase del modelo de salud mental basado en la comunidad (39,2 \% vs. $30 \%$ ). (p>0.05 Test Fisher).

\section{DISCUSIÓN}

La evaluación de los resultados e impacto de las intervenciones en salud constituye un aspecto cada vez más relevante para los formuladores de las políticas en salud. Siempre al evaluar resultados e impacto se plantean dos preguntas : ¿Puede observarse un cambio en las variables definidas? Si es así ¿puede atribuirse el cambio observado a la intervención?. Así mismo, se acepta que el tomar elementos del diseño experimental básico favorece la validez interna de las evaluaciones $(11,12)$. En la presente investigación se tomaron algunos elementos de este tipo de diseño tal como la comparación antes después y entre el grupo estudio y control.

A pesar del anterior reconocimiento la evaluación de modelos de salud mental basados en la comunidad que se han realizado son más de tipo descriptivo, específicamente de proceso y resultado tal como la investigación realizada en Nicaragua entre 1987 y 1990 en la cual con base en el número de consultas psiquiátricas y la conducta terapéutica tomada se estableció una línea de base para posteriormente evaluar el impacto del programa y las modificaciones en conductas diagnósticas y terapéuticas en médicos de atención primaria $(13,14)$.

La OPS también ha señalado otro tipo de experiencias de evaluación de resultado e impacto por ejemplo la realizada en China en la cual se valoró un modelo comunitario de atención de pacientes esquizofrénicos mediante un ensayo clínico al azar “doble ciego” y se evidenció un mejor desempeño social en los pacientes que tenían el abordaje comunitario (20).

En la presente investigación se evalúa los resultados e impactos de la primera fase de un modelo comunitario en el cual a diferencia de los estudios anteriores, la población sujeto no es el enfermo mental sino la población de unas localidades en Bogotá. Esto tiene implicaciones en la formulación de la política pública en salud mental porque orienta las intervenciones más hacia la promoción de la salud que a la prevención $(15,16)$. En la presente investigación se evidenció que en la primera fase del modelo fortaleció las redes de buen trato, las cuales son un determinante de la salud mental.

Se resalta que dos localidades del modelo: Tunjuelito y Antonio Nariño, presentaron un aumento real en la notificación al comparar antes y después del modelo. Esto puede deberse a que en la localidad de Tunjuelito, el hospital tiene la coordinación operativa del modelo, lo cual motiva al equipo de salud y estimula la notificación de casos. Así mismo, las localidades del grupo estudio han tenido mayor disponibilidad de recurso humano para la notificación de casos. Se resalta sin 
embargo, que las mismas dos localidades (Tunjuelito y Antonio Nariño) que habían presentado un mayor aumento en la notificación, también se caracterizan por un mejor seguimiento de estos casos. Las localidades de Tunjuelito y Antonio Nariño son colindantes y se ha evidenciado que los agentes comunitarios en salud formados en Tunjuelito influyeron también en la localidad Antonio Nariño.

De las localidades del grupo estudio, también Tunjuelito y Antonio Nariño presentaron una mejor conectividad con relación a las otras localidades del modelo. La información cualitativa sugiere que la red de buen trato de Tunjuelito ha trabajado en conjunto con la red de buen trato de Antonio Nariño, lo cual puede favorecer una mejor capacidad de estas redes para captar y vincular nuevos nodos.

Así mismo, estas dos localidades son las que presentan mayor crecimiento en nodos de tipo comunitario.

La evaluación sugiere que existe relación entre una mejor conectividad de la red de buen trato, manifestada por mayor número de nodos, mayor composición comunitaria, y la notificación y seguimiento de casos de violencia intrafamiliar y violencia sexual. Lo anterior se explica porque son procesos interrelacionados en los cuales una mayor notificación y seguimiento se logra con más nodos y de tipo comunitario. Estas localidades han tenido mayor participación en las redes de buen trato de instituciones que no son del sector salud, tales como Bienestar Social del Distrito y comisarías de familia $(17,18)$.

Se consideró inicialmente que la primera fase del modelo de salud mental basado en al comunidad mediante la formación de agentes comunitarios, las visitas y tamizajes domiciliarios y la canalización de casos sintomáticos a la consulta de psiquiatría ,producen una mayor capacidad de notificación de casos de violencia intrafamiliar y abuso sexual, lo cual se explica porque los agentes comunitarios favorecen la identificación y canalización de casos hacia la red de Buen Trato. Al realizar visitas domiciliarias se facilita también la identificación de casos no solo de violencia intrafamiliar y sexual sino también trastornos mentales menores o mayores (19). Sin embargo, durante el primer año la notificación de casos no fue tan alta como se esperaba, lo cual se explica porque esta actividad requiere de una red de apoyo social que la soporte y favorezca una ruta critica. La sola presencia de agentes comunitarios, tamizajes y canalizaciones no aumenta la notificación de casos sino existe un sustrato organizativo como una red de buen trato.

Se entiende también que el modelo de salud mental basado en la comunidad es un proceso que demora en consolidarse al menos tres años y que la presente evaluación de impacto es del primer año, lo cual condujo a que se escogieran más indicadores de tipo resultado que de impacto. Que se evaluará la primera fase pudo haber afectado el estudio porque la conectividad y la formación de redes se consolidan más a mediano plazo que a corto plazo $(17,18)$. La presencia de mayor conectividad en las redes de buen trato se interpretó como un impacto por considerarse como un 
determinante de la salud que es afectado en forma positiva por la primera fase del modelo de salud mental basado en la comunidad $(19,20)$.

Una limitación del estudio viene dada por las unidades de observación, las cuales están constituidas por las localidades completas que conforman el grupo estudio y el grupo control, lo cual se considera un tamaño muy grande y que no permite observar la variabilidad de los elementos. Una sola localidad del alguno de los grupos puede tener el volumen poblacional de una ciudad intermedia del país. Esto indica que para futuras evaluaciones es necesario tener unidades de observación más delimitadas tales como las UPZ (unidades programáticas zonales) o los barrios de la localidad.

La presente investigación resalta la importancia de realizar evaluaciones de resultado e impacto en los proyectos que realiza la Secretaría de Salud de Bogotá, debido a que permite identificar en forma temprana las líneas de base que facilitan la continuidad de la evaluación y permiten la identificación de fortalezas y debilidades que pueden ser ajustadas en el desarrollo de las fases posteriores. En este caso se reconoce la necesidad de seguir fortaleciendo las redes sociales de apoyo como la red de buen trato, lo relevante de tener como entrada un eje problémico como la notificación y seguimiento de la violencia intrafamiliar y sexual; y la estructuración de las redes desde sectores comunitarios y de entidades diferentes a las públicas. Así mismo, la presente investigación evidencia que el disponer por parte del hospital público de equipo humano permanente y motivado facilita el trabajo en red.

Agradecimientos: Al equipo de profesionales del modelo comunitario en salud mental del Hospital Tunjuelito-UPA San Benito y a la psiquiatra Diana Alvis de la Secretaria Distrital de Salud.

\section{REFERENCIAS}

1. Polit D, Hungler B. Investigación Científica en ciencias de la Salud. Sexta Edición. México D.F: Mc Graw Hill; 2001:171-209.

2. Banco Internacional de Reconstrucción y Fomento /Banco Mundial. Directivas de Desarrollo. Evaluación del impacto de los proyectos de desarrollo en la pobreza Manual para profesionales, Washington , D.C; 2000: 7-15.

3. OPS/OMS Manual de Monitoreo de la Ejecución de Proyectos; 1999.

4. Martínez -Navarro F, Antó J, Castellanos P. Salud Pública. México D.F: Mc Graw Hill; 1998. p. 363-382.

5. Fundación Santa Fe. Investigación Operativa Aplicada a los Servicios de Salud; 1990. p. 1-5.

OPS. Ensayos de Campo de Intervenciones en Salud en Países en Desarrollo. Washington; 1998. p.9-34.

6. Briones G. Evaluación de Programas Sociales. Editorial Trillas; 1991. p.11-29.

7. Scott- Samuel A, Birley M, Adern K. The Merseyside Guidelines for Health Impact Assessment. Second Edition; 2001.

8. Castro G, Chaves P. Metodología de Evaluación de Impacto de los Proyectos Sociales, UNESCO, Caracas; 1994. p. 11-28. 
9. Desviat F. Salud Mental Comunitaria como estrategia o utopía. Psiquiatría Pública. 2000; 12(1).

10. Campbell D, Stanley J. Diseños Experimentales y Cuasiexperimentales en Investigación Social. Amorrortu Editores. Buenos Aires; 1978. p. 70-74.

11. Levav I. Temas de Salud Mental en la comunidad. Serie PALTEX para Ejecutores de Programas de Salud No, 19. Washington, DC: Organización Panamericana de la Salud; 1992. p. 56-73.

Prieto A. Salud Mental: Situación y Tendencias. Rev. Salud Pública. 2002; 4(1): 74-88

13. Organización Panamericana de la Salud - Organización Mundial de la Salud. Atención en Salud Mental en el Nivel primario: estudio de una muestra de casos en los países centroamericanos; 1990 - 1991.

14. March JC. Los Métodos de Investigación y Evaluación en la Promoción de la Salud. Salud Pública. Mc Graw Hill; 1998.

15. Edwards V, Tapia G. Redes desde la Sociedad Civil: Propuestas para su Potenciación. En Revista Latinoamericana de Educación y Políticas CEAAL, Santiago de Chile; 2 semestre de 1999.

16. OPS/OMS. Programa Mujer, Salud y Desarrollo, La Ruta Crítica de las Mujeres afectadas por la Violencia Intrafamiliar en América Latina, Estudios de Caso en Diez Países. Washington, DC; 2000.

17. Secretaría Distrital de Salud. Política de Salud Mental; 2002.

18. Desjarlais R, Eisenberg L, Good B. Salud Mental en el Mundo. OPS. Problemas y Prioridades en Poblaciones de Bajos Ingresos. Washington, DC;1997. p. 328-243. 